\title{
Coagulation factors in the airways in moderate and severe asthma and the effect of inhaled steroids
}

\author{
F J H Brims, ${ }^{1}$ A J Chauhan, ${ }^{1}$ B Higgins, ${ }^{2}$ J K Shute ${ }^{2}$
}

\begin{abstract}
- An additional appendix is published online only at http:// thorax.bmj.com/content/vol64/ issue12

${ }^{1}$ Respiratory Centre, Queen Alexandra Hospital, Portsmouth Hospitals NHS Trust, UK;

${ }^{2}$ Institute of Biomedical and

Biomolecular Sciences,

University of Portsmouth, UK

Correspondence to:

Dr J Shute, Institute of

Biomedical and Biomolecular

Sciences, St Michael's Building,

White Swan Road, Portsmouth

P01 2DT, UK; jan.shute@port.

ac.uk
\end{abstract}

Received 11 February 2009

Accepted 24 July 2009

Published Online First

23 August 2009

\begin{abstract}
Background: There is evidence of activation of the extrinsic coagulation cascade in the asthmatic airway, and both plasma and locally derived factors may be involved. The hypothesis that the normal haemostatic balance of healthy airways sampled by sputum induction favours fibrin formation in asthmatic airways, and that inhaled corticosteroids (ICS) and plasma exudation influence this balance, was tested.
\end{abstract}

Methods: ELISA and activity assays were used to measure $\alpha_{2}$-macroglobulin (an index of plasma leakage) and coagulation factors in hypertonic saline-induced sputum of 30 stable subjects (10 controls, 10 with moderate asthma and 10 with severe asthma). Additionally, the moderate cohort were weaned off their ICS, followed by further sputum induction 5 days after cessation of steroids.

Results: ICS wean induced a significant rise in plasminogen (median (interquartile range (IOR)): 13.92 (6.12-16.17) vs $4.82(2.14-13.32) \mathrm{ng} / \mathrm{ml} ; 95 \% \mathrm{Cl} 0.003$ to $8.596, p=0.0499$ ) and tissue plasminogen activator (tPA; 5.57 (3.57-14.35) vs 3.88 (1.74-4.05) ng/ml; 95\% $\mathrm{Cl} 0.828$ to $9.972, \mathrm{p}=0.0261$ ) levels in sputum, such that IPA in untreated moderate asthma was significantly $(p=0.0029)$ higher than normal $(2.14(0.0-2.53) \mathrm{ng} / \mathrm{ml})$. Subjects with severe asthma had significantly more $\alpha_{2}$ macroglobulin $(p=0.0003)$, tissue factor $(p=0.023)$, plasminogen activator inhibitor $(p=0.0091)$, thrombinactivatable fibrinolysis inhibitor $(p=0.0031)$ and fibrin degradation products $(p=0.0293)$ in their sputum than control subjects.

Conclusion: Untreated moderate asthma is associated with increased fibrinolysis that is corrected by ICS. Severe asthma and high dose corticosteroid therapy is associated with a profibrinogenic, antifibrinolytic environment in the airways. This study suggests that inhibition of fibrin deposition in severe asthma may be a therapeutic approach.

Asthma is characterised by airway inflammation and variable tissue remodelling. ${ }^{1}$ It is influenced by inhaled corticosteroid (ICS) therapy, yet many of those with asthma remain symptomatic, some with severe manifestations of the disease, as structural changes lead to airflow obstruction that may be irreversible. ${ }^{2}$ Plasma exudation from the bronchial microvasculature contributes to bronchial obstruction by fibrin deposition in mucus plugs, bronchial wall thickening, epithelial shedding, thickening of the basement membrane, hypertrophy of the smooth muscle and mucus secretion. Fibrin deposition in distal airways is also associated with increased bronchial hyper-responsiveness. ${ }^{3}$ We further reported that fibrin formation by bronchial epithelial cells in vitro is independent of plasma proteins, and is essential for bronchial epithelial repair. ${ }^{4}$

Exposure of plasma to cell-bound tissue factor $(\mathrm{TF})$, the principal activator of the extrinsic coagulation cascade, initiates fibrin clot formation within minutes, although the rate of fibrin formation is determined by components of the intrinsic coagulation cascade. ${ }^{5}$ Thus, early, physiologically desirable, fibrin formation for normal wound healing, initiated by TF-bearing cells, may propagate into exudated bulk plasma, generating excessive and physiologically undesirable fibrin that could lead to fibrosis, mucus plug formation, airway narrowing and bronchial hyper-reactivity (BHR). ${ }^{36-8}$

Levels of TF in induced sputum are higher in patients with mild asthma compared with healthy controls, ${ }^{9}$ and after allergen challenge. ${ }^{10}$ A TFdependent increase in coagulation and a plasminogen activator inhibitor (PAI-1)-dependent decrease in fibrinolysis is a feature of alveolar fibrin formation in acute respiratory distress syndrome (ARDS) and idiopathic pulmonary fibrosis. ${ }^{11}$ There has been recent interest in the role of platelets contributing to the coagulation response in patients with acute lung injury ${ }^{12}$; however, their role in the regulation of the extrinsic coagulation cascade in the lung, and in those with asthma is not known. Activation of the coagulation cascade has both fibrin-dependent and fibrin-independent influences on tissue fibrosis. Fibrin-dependent effects include neutralisation of surfactant, binding and accumulation of transforming growth factor $\beta$ (TGF $\beta)$ and provision of a matrix for fibroblast proliferation. Independent effects include activation of serine proteases, notably thrombin, factor VIIa (FVIIa) and FXa, that activate proteaseactivated receptors (PARs), present on fibroblasts and bronchial epithelial cells, to stimulate fibrotic and inflammatory processes further in the lung ${ }^{611} 1_{13}$ and also to enhance TF expression further. ${ }^{14}$ Thus increased levels of TF are selfamplifying when coagulation is initiated.

In the normal lung, the haemostatic balance is antithrombotic and favours fibrinolysis. ${ }^{6} \mathrm{We}$ sought to test the hypothesis that the normal haemostatic balance changes in favour of coagulation in moderate and severe asthma, and that ICS and plasma exudation influence this balance.

\section{METHODS}

\section{Patients}

Healthy controls and patients with moderate and severe asthma were recruited from the community and from the asthma clinic at Queen Alexandra Hospital, Portsmouth, UK. Asthma was confirmed 
by objective assessments of variable airflow obstruction and/or $\mathrm{BHR}$, and together with lung function and exhaled nitric oxide measurements (FeNO), these are described in the online appendix. Subjects were 18-70 years old and had stable symptoms with no need for increased asthma treatment within 6 weeks of study entry. Current smokers, subjects with a smoking history of $>10$ pack years, other coexisting lung disease or significant comorbidity were excluded. "Moderate" asthma was defined as mild persistent disease with low or medium daily dose ICS (200$1000 \mu \mathrm{g}$ beclomethasone dipropionate (BDP) or equivalent) under the Global Initiative for Asthma (GINA) criteria, ${ }^{15}$ with additional asthma medications permitted. "Severe" asthma was defined as GINA severe persistent disease with high daily dose ICS (> $1000 \mu \mathrm{g}$ of BDP or equivalent), with additional asthma drugs (including oral corticosteroids) permitted.

\section{Study design}

After initial screening and 2 week run in, patients underwent induced sputum and venous blood sampling. Furthermore, "moderate" subjects underwent tapered ICS withdrawal (200 $\mu \mathrm{g}$ BDP equivalent per week, see online appendix) 1 week after the first sputum induction. Continuation of long-acting $\beta_{2}$ adrenoceptor agonists (LABAs) and reliever medications was permitted. Subjects completed twice-daily peak expiratory flow (PEF) (Wright mini peak flow meter, Clement Clark International, Harlow, UK) and diary card monitoring with weekly review until 5 days after complete steroid cessation when a second sputum induction was performed.

Ethical approval was granted by the Reading \& Berkshire Research Ethics Committee, and all subjects provided informed consent. Sample size calculations are discussed in the online appendix.

\section{Sputum induction and processing}

Sputum induction was performed using an ultrasonic nebuliser (Devilbiss Ultraneb 2000, Sunrise Medical, UK) and hypertonic saline, and processed with $0.05 \%(\mathrm{w} / \mathrm{v})$ (final concentration) dithiothreitol (DTT), as described in the online appendix.

\section{$\alpha_{2}$-Macroglobulin and coagulation factors in induced sputum}

$\alpha_{2}$-Macroglobulin was used as a marker of plasma exudation ${ }^{16} 17$ and assayed by an in-house ELISA described in the online appendix. TF, tissue factor pathway inhibitor (TFPI), tissue plasminogen activator (tPA) and plasminogen activator inhibitor-1 (PAI-1) were analysed by sandwich ELISA using commercially available kits (American Diagnostica, Stamford, Connecticut, USA). Fibrinogen, FVII, FX, FXIII, plasminogen and thrombin-activatable fibrinolysis inhibitor (TAFI) were analysed by enzyme immunoassay using antibody pairs according to the manufacturer's instructions (Affinity Biologicals, Ancaster, ON, Canada). D-dimers were assayed by sandwich ELISA (Hyphen Biomed, Andresy, France). Fibrin degradation products (FDPs) were measured using the Thrombo-Wellcotest (Remel Europe, Dartford, UK). Enzyme activity for thrombin and plasmin was analysed using specific substrates, S2238 and S2251, respectively (Chromogenix, Milan, Italy). Quantification was with the values for normal reference plasma (Precision Biologic, Dartmouth, NS, Canada). All standards were prepared with $0.05 \%$ (w/v) DTT.

\section{Statistical analyses}

The Kruskal-Wallis test compared median levels across the three independent subgroups. Comparisons between subgroups were made using a Mann-Whitney U test, and 95\% CIs for the difference between medians calculated. Repeated measurements on the moderate asthma cohort were analysed using a paired $t$ test. Associations between parameters were analysed using a pooled $r$ value from the Spearman rank correlation coefficient after heterogeneity testing with a $\chi^{2}$ test. Analysis was performed using GraphPad Prism version 4.03 (GraphPad software, San Diego California, USA).

\section{RESULTS}

Baseline patient characteristics are shown in table 1 . In the moderate group, one patient failed the second sputum induction (after withdrawal of steroids); therefore, nine participants remained in the moderate untreated group, with nine paired sputum samples (presteroid and poststeroid withdrawal) available for analysis.

\section{Blood and airway inflammation}

Sputum differential cell counts are presented in table 2 and blood results in online appendix table 1 . Median values for sputum and blood eosinophilia were significantly higher in the severe group compared with the control group (sputum, 11.2 vs $0.0,95 \%$ CI 1.32 to $31.24, p=0.0001$; blood, 0.6 vs $0.1,95 \%$ CI 0.10 to $0.70, p=0.0095)$. Patients with moderate treated asthma had significantly greater sputum neutrophilia than those with severe asthma (51.8 vs 18.2 , 95\% CI 10.0 to 45.6 , $\mathrm{p}=0.0054$ ). ICS wean resulted in a non-significant increase in the proportion of sputum eosinophils relative to prewean values ( 1.97 vs $0.62,95 \%$ CI -6.11 to $4.91, p=0.404)$, but a significant sputum eosinophilia compared with controls (1.97 vs $0.00,95 \%$ CI 0.76 to $10.4, p=0.0015)$. FeNO levels did not differ between the groups and were not influenced by withdrawal of ICS (18 vs $28.5,95 \%$ CI 3.30 to $29.5, p=0.104$ : online appendix table 1 ).

Sputum $\alpha_{2}$-macroglobulin levels were significantly higher in the severe cohort compared with the other groups (control, 95\% CI 741.3 to 4447.7, $\mathrm{p}=0.0003$; moderate treated, 95\% CI 884.3 to 4676.0, p =0.0002: fig 1 and online appendix table 2) and were not affected by ICS withdrawal (fig 1 and online appendix table 3). Over all groups there was a significant positive correlation with sputum eosinophils $(r=0.676, p<0.001$ : online appendix table 4).

Comparison of $\alpha_{2}$-macroglobulin levels in induced sputum with levels in reference normal plasma $(1000 \mu \mathrm{g} / \mathrm{ml})$ allowed a plasma dilution factor for the samples to be calculated. Thus, plasma proteins were present in induced sputum as a percentage of normal reference values that was: (mean (SD)) controls $0.031 \%(0.021 \%)$, moderate treated $0.015 \%(0.013 \%)$, moderate untreated $0.068 \%(0.135 \%)$ and severe $0.38 \% \quad(0.45 \%)$. Knowledge of these values allowed us to consider the provenance, either plasma exudation or local cellular synthesis, of the coagulation factors present in the airways.

\section{The extrinsic coagulation cascade}

The full results for all assays are provided in online appendix tables 2 and 3 , with a relevant summary provided below.

\section{Fibrinogenesis: TF, FVII and FX}

TF (fig 2A) levels were significantly higher in the severe cohort compared with controls ( 11.85 vs $3.95,95 \%$ CI 1.49 to 16.05 , $\mathrm{p}=0.023)$ and treated moderate asthma (11.85 vs $5.15,95 \% \mathrm{CI}$ 1.3 to $16.21, p=0.0167)$, with no significant difference after weaning ICS (5.915 vs $4.02,95 \%$ CI -9.72 to $2.44, p=0.102$ ). 
Table 1 Patient characteristics

\begin{tabular}{|c|c|c|c|}
\hline & \multicolumn{3}{|l|}{ Group } \\
\hline & Control & Moderate & Severe \\
\hline No. of subjects & 10 & 10 & 10 \\
\hline $\operatorname{Sex}(M / F)$ & $6 / 4$ & $3 / 7$ & $5 / 5$ \\
\hline Age (years) & $36.0(29.5-49.0)$ & $56.0(39.0-62.0)$ & $54.0(42.5-53.5)$ \\
\hline \multicolumn{4}{|l|}{ Smoking history: } \\
\hline Ex & 0 & 2 & 5 \\
\hline Never* & 10 & 8 & 5 \\
\hline Pack years & 0 & 0.75 & 2.1 \\
\hline Atopy $(+/-)^{*}$ & $1 / 9$ & $5 / 5$ & $8 / 2$ \\
\hline ICS dose (BDP equivalent, $\mu \mathrm{g}) \dagger$ & - & $400(200-400)$ & $2000(1800-4000)$ \\
\hline Oral prednisolone (mg daily) & - & - & $4.0(0-7.5)$ \\
\hline \multicolumn{4}{|l|}{ Additional medications: } \\
\hline Long-acting $\beta$-agonist & - & 6 & 8 \\
\hline Leukotriene receptor antagonist & - & 1 & 3 \\
\hline Long-acting antimuscarinic & - & - & 3 \\
\hline Oral theophyline & - & - & 6 \\
\hline Oral antihistamine & - & - & 4 \\
\hline $\mathrm{FEV}_{1} \%$ predicted $\%$ & $103(92-117)$ & 90 (87-108) & $60.5(55-79)$ \\
\hline $\mathrm{FEV}_{1} / \mathrm{FVC}$ ratio & $0.78(0.71-0.82)$ & $0.76(0.74-0.8)$ & $0.62(0.53-0.75)$ \\
\hline Baseline FeNO (ppb) & $15.5(9-26)$ & $18(13-24)$ & $21(8.5-42)$ \\
\hline
\end{tabular}

TF was significantly and positively correlated with sputum eosinophil counts $(r=0.550, p=0.008)$.

FVII (fig 2B) levels were significantly higher in controls compared with the severe cohort ( 2.05 vs $0.00,95 \%$ CI 0.091 to $3.043, p=0.0235)$ and those with untreated moderate asthma ( 2.05 vs $0.07,95 \%$ CI 0.297 to $3.061, p=0.0029$ ). There was a significant reduction in FVII levels following withdrawal of ICS (1.14 vs $0.07,95 \%$ CI 0.10 to 4.697, $p=0.0429$ : fig $2 \mathrm{~B}$, online appendix table 3 ). FX (fig $2 \mathrm{C}$ ) levels were significantly decreased in severe asthma compared with controls (0.910 vs $6.00,95 \%$ CI 0.65 to 9.46, $p=0.0058)$, with no effect following ICS withdrawal (2.62 vs $3.43,95 \%$ CI -6.71 to $6.03, p=0.451)$.

Fibrinolysis: plasminogen, tPA, plasmin, PAI-1, TAFI and FDPs Plasminogen (fig 3A) was significantly lower in treated moderate asthma compared with controls (4.82 vs $12.99,95 \%$ CI 2.62 to $13.62, p=0.0115)$ and ICS withdrawal resulted in a significant increase in levels (4.82 vs 13.92 , 95\% CI 0.003 to 8.596, $p=0.0499$ ).

Levels of tPA (fig 3B) were significantly higher in severe and untreated asthma cohorts when compared with controls (6.26 vs $2.14,95 \%$ CI 1.599 to $11.975, p=0.0046$; and 5.57 vs 2.14 , $95 \%$ CI 1.907 to $10.831, p=0.0015$, respectively), and there was a significant increase in levels following ICS withdrawal (3.88 vs $5.57,95 \%$ CI 0.828 to $9.972, p=0.0261$ : online appendix table 3).

Significantly higher levels of PAI-1, TAFI and FDPs (fig 3C,D,F) were observed in severe asthma compared with controls (95\% CI 1.12 to $12.77, p=0.0091 ; 0.450$ to 6.751 , $\mathrm{p}=0.0031$; and 0.01 to 30.1, $\mathrm{p}=0.0301$, respectively). Furthermore, TAFI was significantly higher in the severe cohort as compared with those with treated asthma (95\% CI 0.852 to $8.049, p=0.0052)$. In the severe group, FDPs levels correlated positively with sputum eosinophil count $(r=0.667, p=0.003)$ and $\mathrm{tPA}(\mathrm{r}=0.493, \mathrm{p}=0.006)$.

Table 2 Sputum differential inflammatory cell counts

\begin{tabular}{|c|c|c|c|c|}
\hline \multirow[b]{3}{*}{ Parameter } & \multicolumn{4}{|l|}{ Group } \\
\hline & Control & Moderate treated & Moderate untreated & Severe \\
\hline & $n=10$ & $n=10$ & $n=9$ & $n=10$ \\
\hline Sputum weight $(\mathrm{g})$ & $3.125(1.72-5.14)$ & $3.04(1.53-3.54)$ & $2.94(0.77-2.69)$ & $2.68(1.54-6.32)$ \\
\hline Cells $/ g$ of sputum $\left(\times 10^{6} / \mathrm{g}\right)$ & $0.76(0.51-2.18)$ & $0.57(0.28-1.88)$ & $1.58(0.51-2.76)$ & $1.13(0.40-3.2)$ \\
\hline Squamous cells (\%) & 16.28 (8.35-28.69) & $19.8(10.3-31.7)$ & $12.0(9.83-22.8)$ & 13.8 (4.59-21.7) \\
\hline Macrophages (\%) & $49.65(23.02-70.11)$ & $43.1(35.5-58.5)$ & $39.9(29.7-66.5)$ & $52.6(28.1-73.6)$ \\
\hline Neutrophils $(\%)^{*}$ & $35.42(18.38-65.43)$ & $51.8(29.2-64.9)$ & $45.4(24.7-59.7)$ & $18.2(12.0-43.1)$ \\
\hline Eosinophils (\%) + \$ & $0.0(0.0-0.36)$ & $0.62(0.28-5.58)$ & $1.97(0.93-11.8)$ & $11.2(1.25-45.5)$ \\
\hline Lymphocytes (\%) & $1.31(0.45-2.37)$ & $1.26(0.55-1.5)$ & $1.11(0.44-2.0)$ & $1.3(1.07-1.96)$ \\
\hline Epithelial cells (\%) & $3.69(1.46-5.35)$ & 1.07 (0.77-2.69) & $1.38(0.69-2.15)$ & $1.08(0.61-1.68)$ \\
\hline
\end{tabular}

All data are presented as the median (interquartile range). All statistical results are from Mann-Whitney $U$ test.

${ }^{*} \mathrm{p}<0.01$, moderate treated group vs severe group.

$\dagger p<0.001$, control group vs severe group.

$\$ \mathrm{p}<0.01$, control group vs moderate untreated group. 


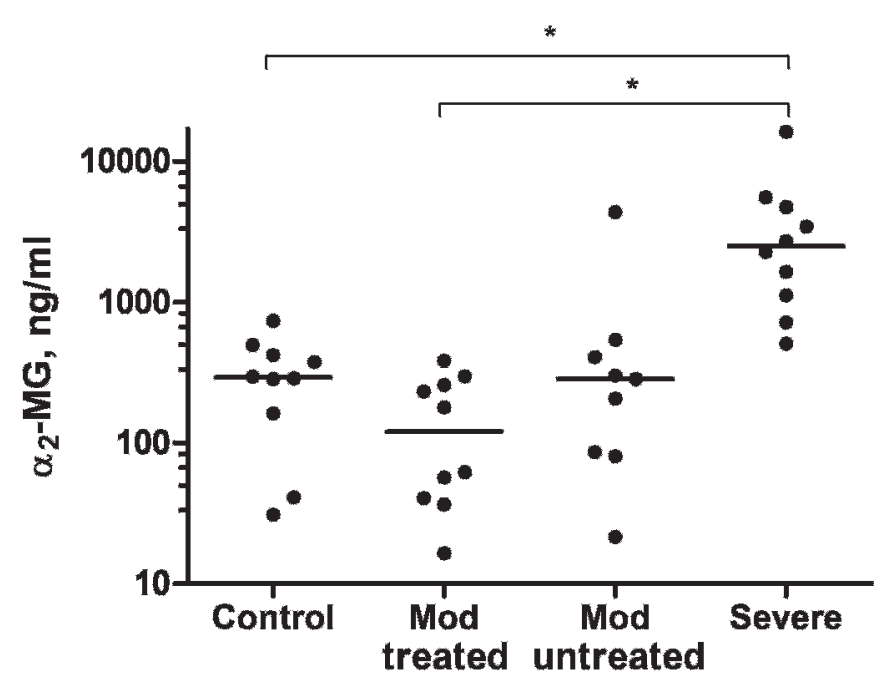

Figure $1 \quad \alpha_{2}$-Macroglobulin ( $\alpha_{2}-M G$ ) levels demonstrating plasma exudation in the airways of controls and subjects with asthma. All data are presented as data points and median with log 10 scale. Mod, moderate. ${ }^{*} \mathrm{p}<0.001$ by Mann-Whitney $\mathrm{U}$ test.

\section{DISCUSSION}

We have compared plasma exudation and coagulation activity in the sputum of healthy controls, patients with moderate persistent asthma, before and after withdrawal of ICS, and patients with severe persistent asthma. ICS withdrawal for 5 days resulted in significantly decreased FVII, increased plasminogen and tPA levels compared with pre-weaning values, and evidence of a more fibrinolytic environment in the airways of patients with untreated moderate asthma compared with controls. Conversely, patients with severe asthma had significantly higher levels of $\alpha_{2}$-macroglobulin, TF, PAI-1 and TAFI compared with those with treated moderate asthma, indicative of a change to a profibrinogenic and antifibrinolytic environment in severe asthma. We believe this is the first study to investigate the haemostatic balance in the airways of patients with severe asthma and following withdrawal of ICS in moderate asthma.

The significantly higher levels of $\alpha_{2}$-macroglobulin in the airways of patients with stable severe asthma are indicative of ongoing bronchial inflammation despite treatment with inhaled and/or oral steroids, and the strong correlation of $\alpha_{2}$-macroglobulin with sputum eosinophils supports this notion.
Figure 2 Profibrinogenic factors in the airways of controls and subjects with asthma. All data are presented as data points and median. Mod, moderate. (A) Tissue factor (TF), (B) factor VII (FVII), (C) factor $X(F X),(D)$ thrombin activity, (E) fibrinogen, (F) factor XIII (FXIII). ${ }^{*} p<0.05$, $\dagger \mathrm{p}<0.01$ both by Mann-Whitney U test; $\$ p<0.05$ by paired t test.
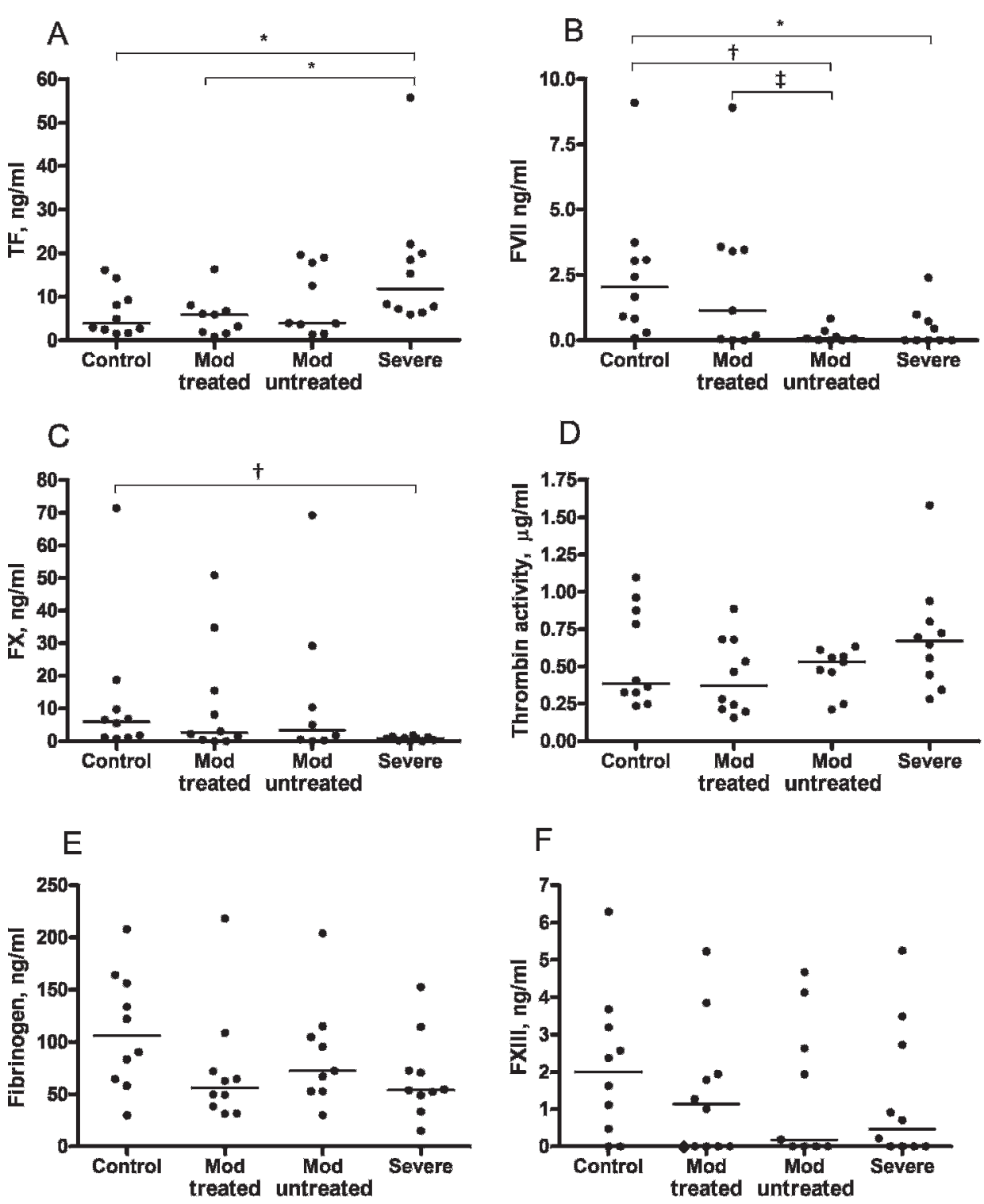
Figure 3 Profibrinolytic and antifibrinolytic factors in the airways of controls and subjects with asthma. All data are presented as data points and median. Mod, moderate. (A) plasminogen, $(B)$ tissue plasminogen activator (tPA), (C) plasminogen activator inhibitor (PAI-1), (D) thrombin-activatable fibrinolysis inhibitor (TAFI), (E) D-dimers ( $\log 10$ scale), (F) fibrin degradation products (FDPs). The dotted line on the FDP graph indicates the lower limit of detection $(<2 \mu \mathrm{g} / \mathrm{ml})$, with log 10 scale; only performed on control and severe cohorts, see online appendix. ${ }^{*} p<0.05$, $\uparrow \mathrm{p}<0.01$ both by Mann-Whitney U test; $\$ p<0.05$ by paired t test.
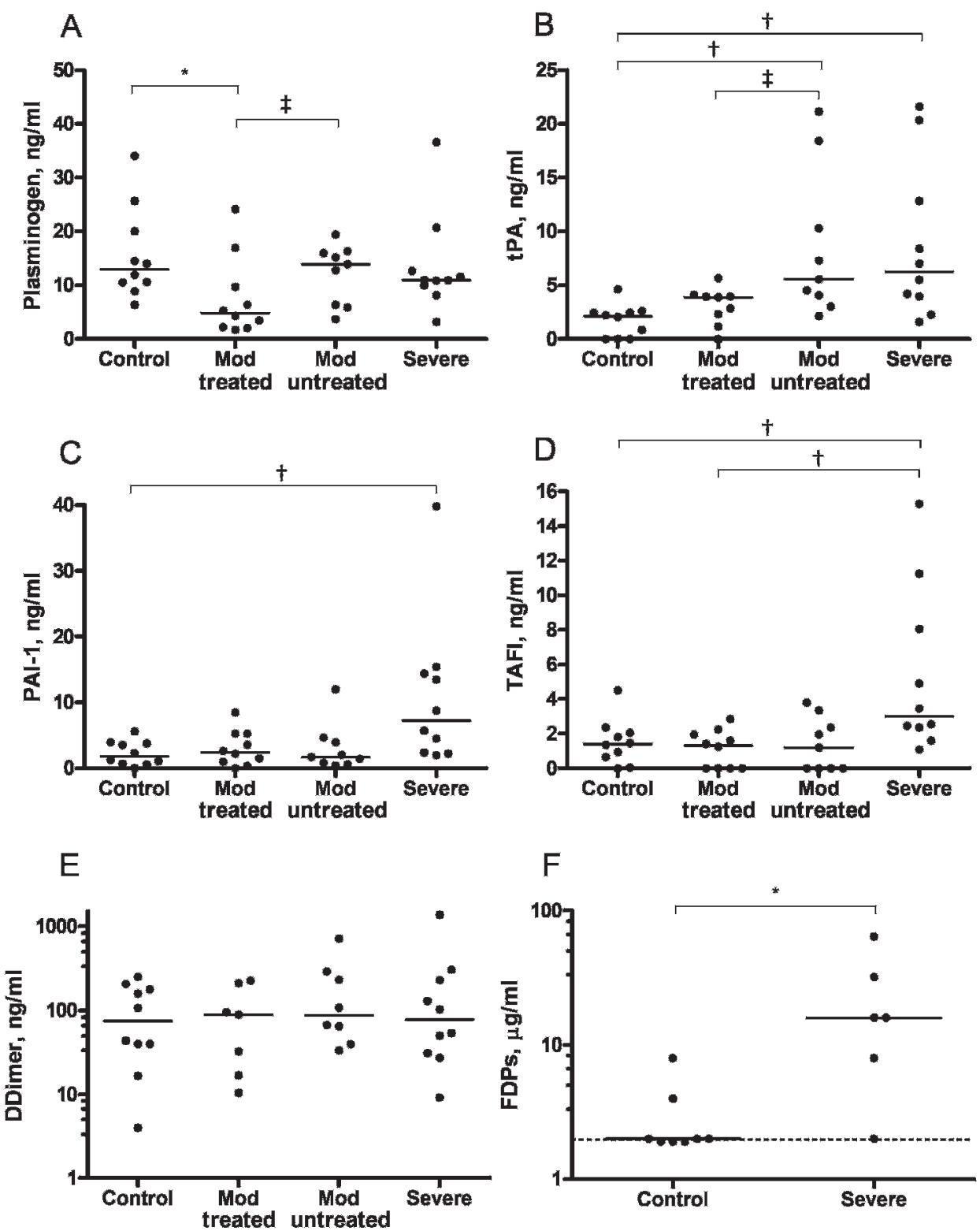

Discussion of the study design, and cohort characteristics, including sputum differential cell counts and FeNO measurements, is provided in the online appendix.

\section{Fibrinogenesis}

The higher levels of TF in the airways of patients with severe asthma, in the absence of detectable difference in TFPI levels, indicates a potentially procoagulant environment. Taking into account the plasma dilution factor, most of the TF we detected in the airways is likely to derive from local cellular sources, including monocytes, macrophages, ${ }^{18}$ eosinophils ${ }^{19}$ and possibly apoptotic bronchial epithelial cells ${ }^{20}$ that are features of severe asthma.

Levels of FVII were significantly lower in both untreated and severe asthma. This raises the possibility that FVII may be reduced by a mechanism associated with asthma pathogenesis, rather than therapy. Our unpublished in vitro evidence indicates that FVII and FX rapidly bind to bronchial epithelial cells, supporting the notion that these factors are lower in severe asthma because coagulation is initiated on cell surfaces by TF via reactions dependent on catalytic phospholipids in the cell membranes, and our samples have examined supernatant, not cell-bound FVII or FX. A strong negative correlation of these factors with observed TF levels in this study (FVII, $r=-0.610$, $p=0.002 ; F X, r=-0.634, p=0.001$ ) lends further support for this hypothesis. Therefore, our observation may reflect binding and consumption of FVII and FX in the coagulation process, rather than their absence, which would completely abolish coagulation. Whilst not measured in this current study, levels of FXa activity have been shown to correlate with mucosal thickness and collagen deposition in a mouse model of asthma, and blockade with FXa inhibitor reduced airway hyperresponsiveness. ${ }^{21}$

Thrombin activity is normally undetectable in plasma, but was detected in all sputum samples (fig 2D), indicating local activation of prothrombin in the airways. Considering the plasma dilution factor, thrombin in the healthy airway is likely to be largely (94\%) derived from locally expressed prothrombin, activated by FXa. However, prothrombin expression by bronchial epithelial cells remains to be confirmed by reverse transcription-PCR.

Levels of fibrinogen in our controls and subjects with asthma were similar to those reported elsewhere, ${ }^{22}{ }^{23}$ although this is the 
first study to report levels in stable severe asthma and a positive correlation of fibrinogen with D-dimers $(r=0.417, p=0.050)$ was observed. The levels in the control group may equate to derivation by plasma exudation, although a local source is not ruled out. ${ }^{4}$ However, values in the asthma groups were lower than those that would be derived from unfiltered plasma, indicating that fibrinogen is either deposited in the tissue matrix before it arrives at the epithelium ${ }^{24}$ or rapidly cleaved by thrombin and deposited as insoluble fibrin on epithelial cell surfaces.

FXIII levels in the severe group $(0.46(0.0-3.1) \mathrm{ng} / \mathrm{ml})$ were lower than predicted $(\sim 38 \mathrm{ng} / \mathrm{ml})$ by the plasma dilution factor. This indicates that FXIII may be adsorbed by the fibrin clot that forms as FXIII cross-links fibrin monomers, and/or that FXIII is proteolytically degraded, for example by neutrophil elastase which is shown to regulate FXIII within fibrin clots. ${ }^{25}$

\section{Fibrinolysis}

tPA is expressed by a number of cells in the airway including mast cells, macrophages, fibroblasts ${ }^{7}$ and bronchial epithelial cells. ${ }^{26} \mathrm{We}$ have demonstrated higher levels of tPA in the severe and untreated asthma groups, which may indicate a disease-related increase in tPA expression. D-dimers were detected in all samples, indicating fibrin formation and fibrinolysis even in normal healthy airways, as previously reported. ${ }^{4}{ }^{27}$ Despite significantly higher levels of tPA in untreated moderate and severe asthma, there were no significant differences in D-dimer levels between the groups which may have been degraded by local neutrophil elastase activity. ${ }^{28}$ Fibrinolysis is negatively regulated by PAI- 1 and TAFI, both of which were significantly higher in the severe group as compared with controls, and calculation of a PAI-1/tPA molar ratio demonstrated a raised ratio in the severe cohort (control group median 0.873 , severe 1.455 ) which suggests an antifibrinolytic environment in the airways, related to asthma severity. The higher FDP levels in severe subjects compared with controls is likely to represent overall upregulation of the external coagulation cascade with fibrin accumulation.

Levels of plasminogen detected in sputum are lower than those predicted from plasma dilution, indicative of binding of plasminogen to fibrin and cell surfaces, which accelerates its conversion to plasmin by tPA. Plasminogen expression by cells outside of the liver, including keratinocytes (reviewed by Irigoyen et $a^{29}$ ), has been reported, but to our knowledge there are currently no reports of plasminogen expression by cells in the airway. Concentrations of tPA and PAI- 1 in induced sputum are orders of magnitude higher than predicted from plasma exudation alone, and most must come from local cellular sources. TAFI was detected at levels that could be expected from plasma exudation, and TAFI expression by cells other than hepatocytes and adipocytes has not been described. ${ }^{30}$

Neutrophils, ${ }^{31}$ mast cells, ${ }^{8}$ macrophages, fibroblasts and the bronchial epithelium ${ }^{7}$ are potential local sources of PAI-1. A 4G/ 5G polymorphism in the PAI-1 promoter region has been reported, and preferential transmission of the 4G allele correlates with increased PAI-1 expression in asthma. ${ }^{8} 32$ PAI-1 and TAFI have been implicated in normal wound healing, and increased expression of both PAI- $1^{78}$ and TAFI ${ }^{30}{ }^{33}$ has also been implicated in fibrin formation leading to the development of lung fibrosis. To our knowledge this is the first report of increased TAFI levels in the asthmatic airway.

\section{The role of corticosteroids}

Plasminogen levels were significantly lower in those with treated moderate asthma compared with controls and rose significantly on cessation of ICS, with no change in plasma exudation, indicating that ICS may suppress local plasminogen expression. A similar suppressive effect of ICS on local tPA expression, possibly by bronchial epithelial cells, ${ }^{34}$ was demonstrated in the same group. Lower plasminogen and tPA levels would tend to reduce fibrinolysis and support fibrin-dependent epithelial repair in the airway, revealing a possible mechanism by which ICS contribute to epithelial continuity in moderate asthma.

PAI-1 and TAFI expression is enhanced by corticosteroids, ${ }^{30}{ }^{35}$ and when oral and inhaled corticosteroid doses are converted to equivalent units (see online appendix) a significant correlation of PAI- 1 with corticosteroid dose $(r=0.46, p=0.04)$ was found for the moderate and severe groups. This raises the possibility that corticosteroids contribute to the increased expression of PAI-1 and TAFI in severe asthma and excessive fibrin formation that may be contributing to airway narrowing and $\mathrm{BHR} .^{36-8}$

\section{Conclusions}

Defective fibrin formation and/or instability, and increased degradation of the fibrin matrix may contribute to the pathogenesis of moderate asthma, an effect that can be reversed by treatment with ICS.

Conversely, the levels of coagulation factors in severe asthma indicate a procoagulant, antifibrinolytic environment in which excessive fibrin formation and accumulation may contribute to irreversible airway narrowing and subepithelial bronchial fibrosis. Since PAI-1 and TAFI control the clearance of fibrin in the lung, and a stimulatory effect of PAI-1 on neutrophilic inflammation has been reported, ${ }^{31}{ }^{36}$ our findings suggest that these molecules may be possible therapeutic targets in severe asthma.

Funding: Defence Postgraduate Medical Deanery (DPMD), Birmingham, UK. We wish to acknowledge the support of Asthma UK (Grant Ref 02/048) for our pilot studies of coagulation factors in asthma.

Competing interests: None.

Ethics approval: A favourable local research ethics committee opinion was obtained for the study.

Provenance and peer review: Not commissioned; externally peer reviewed.

\section{REFERENCES}

1. Holgate ST. Pathogenesis of asthma. Clin Exp Allergy 2008;38:872-97.

2. Bergeron C, Boulet LP. Structural changes in airway diseases: characteristics, mechanisms, consequences, and pharmacologic modulation. Chest 2006;129:106887.

3. Wagers SS, Norton RJ, Rinaldi LM, et al. Extravascular fibrin, plasminogen activator, plasminogen activator inhibitors, and airway hyperresponsiveness. J Clin Invest 2004:114:104-11.

4. Perrio MJ, Ewen D, Trevethick MA, et al. Fibrin formation by wounded bronchial epithelial cell layers in vitro is essential for normal epithelial repair and independent of plasma proteins. Clin Exp Allergy 2007;37:1688-700.

5. Ovanesov MV, Ananyeva NM, Panteleev MA, et al. Initiation and propagation of coagulation from tissue factor-bearing cell monolayers to plasma: initiator cells do not regulate spatial growth rate. J Thromb Haemost 2005;3:321-31.

6. Chambers RC. Procoagulant signalling mechanisms in lung inflammation and fibrosis: novel opportunities for pharmacological intervention? Br J Pharmacol 2008;153(Suppl 1):S367-78.

7. Kucharewicz I, Kowal K, Buczko W, et al. The plasmin system in airway remodeling Thromb Res 2003;112:1-7.

8. Cho SH, Ryu $\mathrm{CH}$, Oh CK. Plasminogen activator inhibitor-1 in the pathogenesis of asthma. Exp Biol Med (Maywood) 2004;229:138-46.

9. Gabazza EC, Taguchi 0, Tamaki S, et al. Thrombin in the airways of asthmatic patients. Lung 1999;177:253-62.

10. Terada M, Kelly EA, Jarjour NN. Increased thrombin activity after allergen challenge: a potential link to airway remodeling? Am J Respir Crit Care Med 2004;169:373-7.

11. Wygrecka M, Jablonska E, Guenther A, et al. Current view on alveolar coagulation and fibrinolysis in acute inflammatory and chronic interstitial lung diseases. Thromb Haemost 2008;99:494-501. 
12. Tabuchi A, Kuebler WM. Endothelium-platelet interactions in inflammatory lung disease. Vascul Pharmacol 2008;49:141-50.

13. Krupiczojc MA, Scotton CJ, Chambers RC. Coagulation signalling following tissue injury: focus on the role of factor Xa. Int J Biochem Cell Biol 2008;40:1228-37.

14. Chu AJ. Tissue factor mediates inflammation. Arch Biochem Biophys 2005;440:12332.

15. GINA. Global Strategy for Asthma Management and Prevention. Global Initiative for Asthma (GINA) 2007;Available from: http://www.ginasthma.org.

16. Persson CG, Erjefalt JS, Greiff $\mathrm{L}$, et al. Contribution of plasma-derived molecules to mucosal immune defence, disease and repair in the airways. Scand J Immunol 1998;47:302-13

17. Van Rensen EL, Hiemstra PS, Rabe KF, et al. Assessment of microvascular leakage via sputum induction: the role of substance $P$ and neurokinin $A$ in patients with asthma. Am J Respir Crit Care Med 2002;165:1275-9.

18. Drake TA, Morrissey JH, Edgington TS. Selective cellular expression of tissue factor in human tissues. Implications for disorders of hemostasis and thrombosis. Am J Pathol 1989;134:1087-97.

19. Moosbauer C, Morgenstern E, Cuvelier SL, et al. Eosinophils are a major intravascular location for tissue factor storage and exposure. Blood 2007:109:995-1002.

20. Cohen L, Tarsi J, Ramkumar T, et al. Epithelial cell proliferation contributes to airway remodeling in severe asthma. Am J Respir Crit Care Med 2007:176:138-45.

21. Shinagawa K, Martin JA, Ploplis V, et al. Coagulation factor Xa modulates airway remodeling in a murine model of asthma. Am J Resp Crit Care Med 2007:175:136-43.

22. Pizzichini E, Pizzichini MM, Efthimiadis A, et al. Indices of airway inflammation in induced sputum: reproducibility and validity of cell and fluid-phase measurements. Am J Respir Crit Care Med 1996;154:308-17.

23. Kelly MM, Leigh $\mathrm{R}$, Jayaram $\mathrm{L}$, et al. Eosinophilic bronchitis in asthma: a model for establishing dose-response and relative potency of inhaled corticosteroids. J Allergy Clin Immunol 2006;117:989-94.

24. Guadiz G, Sporn LA, Simpson-Haidaris PJ. Thrombin cleavage-independent deposition of fibrinogen in extracellular matrices. Blood 1997;90:2644-53.
25. Bagoly Z, Haramura G, Muszbek L. Down-regulation of activated factor XIII by polymorphonuclear granulocyte proteases within fibrin clot. Thromb Haemost 2007;98:359-67.

26. Chu EK, Cheng J, Foley JS, et al. Induction of the plasminogen activator system by mechanical stimulation of human bronchial epithelial cells. Am J Respir Cell Mol Biol 2006;35:628-38.

27. Katona E, Nagy B, Kappelmayer J, et al. Factor XIII in bronchoalveolar lavage fluid from children with chronic bronchoalveolar inflammation. J Thromb Haemost 2005;3:1407-13.

28. Bach-Gansmo ET, Halvorsen S, Godal HC, et al. D-dimers are degraded by human neutrophil elastase. Thromb Res 1996;82:177-86.

29. Irigoyen JP, Munoz-Canoves P, Montero L, et al. The plasminogen activator system: biology and regulation. Cell Mol Life Sci 1999;56:104-32.

30. Boffa MB, Koschinsky ML. Curiouser and curiouser: recent advances in measurement of thrombin-activatable fibrinolysis inhibitor (TAFI) and in understanding its molecular genetics, gene regulation, and biological roles. Clin Biochem 2007:40:431-42.

31. Xiao W, Hsu YP, Ishizaka A, et al. Sputum cathelicidin, urokinase plasminogen activation system components, and cytokines discriminate cystic fibrosis, COPD, and asthma inflammation. Chest 2005;128:2316-26.

32. Kowal K, Zukowski S, Moniuszko M, et al. Plasminogen activator inhibitor-1 (PAl-1) and urokinase plasminogen activator (UPA) in sputum of allergic asthma patients. Folia Histochem Cytobiol 2008:46:193-8.

33. Fujimoto H, Gabazza EC, Taguchi $\mathbf{0}$, et al. Thrombin-activatable fibrinolysis inhibitor deficiency attenuates bleomycin-induced lung fibrosis. Am J Pathol 2006;168:108696.

34. Myohanen H, Virtanen I, Vaheri A. Elimination of hydrocortisone from the medium enables tissue plasminogen activator gene expression by normal and immortalized nonmalignant human epithelial cells. Biol Chem 2001;382:1563-73.

35. Saksela 0, Rifkin DB. Cell-associated plasminogen activation: regulation and physiological functions. Annu Rev Cell Biol 1988;4:93-126.

36. Marshall LJ, Ramdin LS, Brooks T, et al. Plasminogen activator inhibitor-1 supports IL-8-mediated neutrophil transendothelial migration by inhibition of the constitutive shedding of endothelial IL-8/heparan sulfate/syndecan-1 complexes. J Immunol 2003;171:2057-65

\section{Treatment with low-dose lansoprazole may reduce frequency of COPD exacerbations in older patients}

Colds caused by respiratory viruses can trigger exacerbations of chronic obstructive pulmonary disease (COPD). This Japanese study investigated whether lansoprazole reduces the frequency of COPD exacerbations by mitigating the effects of respiratory viruses.

One hundred and three patients with COPD (mean age 75 years, 95\% male), but without evidence of gastro-oesophageal disease, were randomised to receive lansoprazole $15 \mathrm{mg}$ once daily or no treatment on top of their usual COPD medications. Patients were followed for 1 year during which time they recorded a daily score for symptoms of a common cold and attended hospital fortnightly. The authors used a healthcare utilisation definition for an exacerbation of COPD.

Direct comparison of the two groups using standard parametric tests revealed no difference in the frequency of common colds between the two groups, although multivariate analysis suggested that lansoprazole use may be associated with a lower risk of frequent colds ( $>3 /$ year). There was a significant reduction in COPD exacerbations in the lansoprazole group (mean 0.34 exacerbations per year in the lansoprazole group vs 1.18 in the control group).

Apart from one patient in the treatment arm who withdrew due to diarrhoea, the authors give no information on adverse events in patients treated with lansoprazole. Reduction in viral infection by treatment with lansoprazole certainly warrants further study.

- Sasaki T, Nakayama K, Yasuda H, et al. A randomized, single blind study of lansoprazole for the prevention of exacerbations of chronic obstructive pulmonary disease in older patients. J Am Geriatr Soc 2009;57:1453-7.

\section{Laura Willis}

Correspondence to: Dr L Willis, SpR, Northwick Park Hospital, Harrow, UK; willislk@doctors.org.uk

Thorax 2009;64:1043. doi:10.1136/thx.2009.127431 